\title{
ADSCs and adipocytes are the main producers in the autotaxin- lysophosphatidic acid axis of breast cancer and healthy mammary tissue in vitro
}

Rafael Schmid ${ }^{1 \dagger}$, Katharina Wolf ${ }^{2 \dagger}$, Jan W. Robering ${ }^{1}$, Selina Strauß ${ }^{2}$, Pamela L. Strissel ${ }^{3}$, Reiner Strick ${ }^{3}$, Matthias Rübner ${ }^{3}$, Peter A. Fasching ${ }^{4}$, Raymund E. Horch', Andreas E. Kremer², Anja M. Boos ${ }^{1}$

and Annika Weigand ${ }^{1 *}$ (D)

\begin{abstract}
Background: Breast cancer is the most common malignancy in women affecting one out of eight females throughout their lives. Autotaxin (ATX) is upregulated in breast cancer which results in increased lysophosphatidic acid (LPA) formation within the tumor. This study's aim was to identify the role of different mammary cell populations within the ATX-LPA axis.

Methods: Epithelial-cell-adhesion-molecule-positive (EPCAM) and -negative cells from breast tumors, adiposederived stem cells (ADSCS) of tumor-adjacent and tumor-distant mammary fat were isolated and compared to healthy ADSCs, mammary epithelial cells (HMECs), and mesenchymal cells (MES) of healthy mammary tissue $(n=4$ each) and further to well-established breast (cancer) cell lines.

Results: mRNA expression analyses revealed that ADSCS and MES largely expressed LPA receptor 1 (LPAR1) while epithelial cells mainly expressed LPAR6. LPA 18:1 activated all the cell populations and cell lines by rise in cytosolic free calcium concentrations. MES and ADSCs expressed ATX whereas epithelial cells did not. ADSCs revealed the highest expression in ATX with a significant decline after adipogenic differentiation in healthy ADSCs, whereas ATX expression increased in ADSCs from tumor patients. Breast (cancer) cell lines did not express ATX. Transmigration of MES was stimulated by LPA whereas an inhibitory effect was observed in epithelial cells with no differences between tumors and healthy cells. Triple-negative breast cancer (TNBC) cell lines were also stimulated and the transmigration partly inhibited using the LPA receptor antagonist Ki16425.

Conclusions: We here show that each mammary cell population plays a different role in the ATX-LPA axis with ADSCs and adipocytes being the main source of ATX in tumor patients in our experimental setting. Inhibitors of this axis may therefore present a valuable target for pharmacological therapies.
\end{abstract}

Keywords: Breast cancer, Autotaxin, Lysophosphatidic acid, Therapy, ADSC

\footnotetext{
* Correspondence: annika.weigand@uk-erlangen.de

${ }^{+}$Rafael Schmid and Katharina Wolf contributed equally to this work

'Department of Plastic and Hand Surgery and Laboratory for Tissue

Engineering and Regenerative Medicine, University Hospital of Erlangen,

Friedrich-Alexander-University Erlangen-Nürnberg (FAU), Krankenhausstr. 12,

91054 Erlangen, Germany

Full list of author information is available at the end of the article
}

(c) The Author(s). 2018 Open Access This article is distributed under the terms of the Creative Commons Attribution 4.0 International License (http://creativecommons.org/licenses/by/4.0/), which permits unrestricted use, distribution, and reproduction in any medium, provided you give appropriate credit to the original author(s) and the source, provide a link to the Creative Commons license, and indicate if changes were made. The Creative Commons Public Domain Dedication waiver (http://creativecommons.org/publicdomain/zero/1.0/) applies to the data made available in this article, unless otherwise stated. 


\section{Background}

As breast cancer affects one out of eight women throughout their lives, it is the most common malignancy amongst women. Nevertheless, due to improved cancer detection and treatment, mortality has decreased over the past 20 years [1], however, treatments often fail. Metastatic breast tumors tend to develop resistance to treatments with chemotherapeutics or radiotherapy. Current targeted therapies seem to offer significant improvement in some but not all of these cases [2]. Another candidate for target therapies could be autotaxin (ATX), a gene that is among the top 40 most up-regulated genes in metastatic cancer cell lines [3].

ATX was originally identified as autocrine cell motility factor in conditioned medium of melanoma cells [4]. Initially, it was believed to function mainly as pyrophosphatase and was therefore named ectonucleotide pyrophosphatase/phosphodiesterase 2, ENPP2 [5]. Later, its lysophospholipase activity was recognized which is responsible for hydrolyzing lysophosphatidyl choline (LPC) into lysophosphatidic acid (LPA) [6, 7]. LPA is a small bioactive phospholipid which signals via at least six different G-protein coupled receptors entitled LPAR1-6 [810]. The LPA-ATX axis influences several cellular pathways [11] including those concerning cell proliferation and migration especially of tumor cells [12-16]. In recent studies, the stimulatory influence of adipocyte derived ATX $[17,18]$ on breast cancer cell proliferation and breast cancer progression was revealed [19-21].

ATX expression and thereby LPA synthesis is upregulated in invasive breast cancers [22]. Benesch et al. [19] published that the combination of a breast tumor and the surrounding fat tissue leads to a vicious cycle. Increased inflammatory cytokines lead to increased ATX expression and augmented ATX levels within the tumor. The tumor surrounding fat tissue of the breast represents a major source of ATX which is in contrast to other carcinomas such as thyroid cancer [23]. Elevated ATX and LPA levels reduce the capacity of inducing apoptosis in tumor cells, thereby contributing to the resistance to chemotherapeutics and radiotherapy [24]. A recent study of Meng et al. [20] indicated that radiotherapy further increased ATX production in adipose tissue. Further, high levels of the LPAR3 in epithelial cells are also associated with a more aggressive tumor progression [25]. Dysfunctional regulation of the ATXLPA axis, even on the receptor site, could promote the formation of breast tumors. In transgenic mice with overexpression of ATX, LPAR1, LPAR2, or LPAR3, spontaneous mammary tumor formation has been reported [26].

Many findings on ATX in breast cancer are based on tumor cell line models and so it remains unclear which cell populations within the tumor environment secrete
ATX and thereby contribute to the vicious cycle of tumor progression [19]. We here evaluate this model with different primary human mammary-derived cell types of breast cancer patients and compare these results to primary mammary cells of healthy tissue and several breast (cancer) cell lines. This study can help to analyze which cells are the source of elevated ATX levels in breast cancer and clarify tumorous cell-cell interactions as well as it may offer an opportunity for targeted therapies.

\section{Methods}

Isolation of epithelial and mesenchymal tumor cells

For the isolation of epithelial and mesenchymal cells from luminal $\mathrm{B}$ or triple-negative mammary carcinomas (TNBC) ( $n=4$ in total), a human Tumor Dissociation Kit (Miltenyi Biotec GmbH, Bergisch Gladbach, Germany) was used.

The carcinoma was washed ten times with PBS (Sigma-Aldrich, St. Louis, MO, USA). The tumor was cut into small pieces of $2-4 \mathrm{~mm}^{3}$. The kit's enzymes were used according the manufacturer's protocol in DMEM (Biochrom, Berlin, Germany) and put into a gentleMACS C Tube (Miltenyi Biotec $\mathrm{GmbH}$ ) together with the tumor. A gentleMACS ${ }^{\text {min }}$ Octo Dissociator was used to run program 37C_h_TDK_3 followed by program m_imptumor_01 and filtration through a 70- $\mu \mathrm{m}$ MACS SmartStrainer (all from Miltenyi Biotec $\mathrm{GmbH}$ ) according to the manual. The resulting suspension was centrifuged at $300 \mathrm{~g}$ for $7 \mathrm{~min}$. The pellet was resuspended in $300 \mu \mathrm{l}$ MACS buffer $(0.5 \%$ bovine serum albumin [BSA], 2 mM EDTA in PBS adjusted to pH 7.2 [Sigma-Aldrich]) and $100 \mu \mathrm{l}$ human FcR Blocking Reagent as well as $100 \mu \mathrm{l} \mathrm{CD326} \mathrm{(EpCAM)} \mathrm{MicroBeads,} \mathrm{human} \mathrm{(all} \mathrm{from}$ Miltenyi Biotec $\mathrm{GmbH}$ ) were added. The magnetic separation was performed in LS Columns and a MidiMACS $^{\mathrm{Tm}}$ Separator (Miltenyi Biotec $\mathrm{GmbH}$ ) using the MACS buffer. This epithelial-cell-adhesion-molecule-positive (EpCAM) fraction, named epithelial tumor cells (BCC), was centrifuged at $300 \mathrm{~g}$ for $10 \mathrm{~min}$ and seeded in Mammary Epithelial Cell Medium (ScienCell Research Laboratories, Carlsbad, CA, USA) supplemented with $100 \mathrm{U} / \mathrm{ml}$ penicillin and $0.1 \mathrm{mg} / \mathrm{ml}$ streptomycin (Sigma-Aldrich) as well as 5\% FBS Superior (Biochrom) onto collagen-coated $\left(6 \mu \mathrm{g} / \mathrm{cm}^{2}\right.$ rat tail collagen type I [Sigma-Aldrich]) 6-well plates. The medium was changed to medium without FBS after $24 \mathrm{~h}$.

The unlabeled fraction was collected and further sorted using CD31 MicroBeads, human in an MS Column and a MiniMACS $^{\text {tm }}$ Separator (all from Miltenyi Biotec GmbH). The CD31-negative flow through, named mesenchymal tumor cells (MES t) was cultured in collagen-coated $25-\mathrm{cm}^{2}$ tissue culture flasks using the EpiCult $\mathrm{t}^{\mathrm{m}}-\mathrm{C}$ Human Medium Kit (STEMCELL Technologies Germany GmbH, 
Cologne, Germany) supplemented with $2 \mathrm{mML}$-glutamine (Sigma-Aldrich), penicillin-streptomycin $(100 \mathrm{U} / \mathrm{ml}$, $0.1 \mathrm{mg} / \mathrm{ml}), 5 \%$ FBS superior, and $0.48 \mu \mathrm{g} / \mathrm{ml}$ hydrocortisone (STEMCELL Technologies Germany $\mathrm{GmbH}$ ). The first medium change was performed after $48 \mathrm{~h}$. After one week the media for BCC and MES were replaced with media without penicillin-streptomycin.

\section{Isolation of healthy, tumor-adjacent, and tumor-distant adipose-derived stem cells}

Adipose-derived stem cells (ADSCs) were either isolated from healthy tissue (ADSC h, $n=4$ ) or from luminal B or triple-negative mammary carcinoma patients $(n=4$ in total).

Tumor-adjacent ADSCs (ADSC ta) were isolated from fat that was directly surrounding the tumor, tumor distant ADSCs (ADSC td) were from at least $10 \mathrm{~cm}$ distant mammary fat tissue.

Each tissue was cut into small pieces of less than 2 $\mathrm{mm}^{3}$. They were incubated in $20 \mathrm{ml}$ collagenase solution $(0.1 \%$ collagenase I [Biochrom] in PBS) and incubated at $37^{\circ} \mathrm{C}$ for $120 \mathrm{~min}$ on a tube roller. The digestion was stopped using $20 \mathrm{ml}$ Gibco $^{\text {тм }}$ MEM $\alpha$ including GlutaMAX $^{\mathrm{TM}}$ Supplement (Thermo Fisher Scientific Inc., Waltham, MA, USA) and 10\% FBS Superior. This was followed by a centrifugation step ( $400 \mathrm{~g}, 10 \mathrm{~min})$. The pellet was resuspended in MEM $\alpha$ with $10 \%$ FBS and penicillin-streptomycin $(100 \mathrm{U} / \mathrm{ml}, 0.1 \mathrm{mg} / \mathrm{ml})$ and filtered through a $70-\mu \mathrm{m}$ cell strainer. The cells were seeded into $75-\mathrm{cm}^{2}$ cell culture flasks. The first medium change was done after $48 \mathrm{~h}$ and the antibiotics omitted after 1 week.

Isolation of normal mammary epithelial cells (HMEC), normal mesenchymal cells (MES $h$ ) and normal adiposederived stem cells (ADSC $h$ )

For the isolation of normal mammary epithelial cells (HMEC), healthy mammary tissue $(n=4)$, was used, as described previously [27].

Summarized, the mammary tissue was cut into small pieces, followed by enzymatic incubation periods with collagenase, hyaluronidase, and trypsin and further cell fractionation. Epithelial cells were successfully separated with $>95 \%$ purity from mesenchymal cells using this protocol. The patient-matching ADSC $\mathrm{h}$ were isolated from breast fat tissue using collagenase digestion and incubation in red blood cell lysis buffer as previously described [27].

Like BCC and MES t, HMEC and MES h were cultivated in collagen-coated $75-\mathrm{cm}^{2}$ culture flasks, in Mammary Epithelial Cell Medium respectively EpiCult ${ }^{\mathrm{Tm}}-\mathrm{C}$ Human Medium supplemented with 5\% FBS, $2 \mathrm{mM}$ L-glutamine, and hydrocortisone $(0.48 \mu \mathrm{g} / \mathrm{ml})$. ADSC $\mathrm{h}$ were cultivated in MEM $\alpha$ supplemented with $10 \%$ FBS.
Medium was changed every 2-3 days. All cells were incubated at $37{ }^{\circ} \mathrm{C}$ and $5 \% \mathrm{CO}_{2}$. Cells were used in passage $4-8$.

\section{Cell culture of breast (cancer) cell lines}

Different cell lines (all from American Type Culture Collection ATCC, Manassas, VA, USA) were used to compare the results of primarily isolated cells to established models. The mammary epithelial cell line MCF-10A (ATCC CRL-10317 ${ }^{\mathrm{TM}}$ ) was cultivated in Mammary Epithelial Cell Growth Medium (PromoCell GmbH, Heidelberg, Germany) with the addition of $100 \mathrm{ng} / \mathrm{ml}$ cholera toxin (Sigma Aldrich). Furthermore, we used six different breast cancer cell lines. BT-474 (ATCC ${ }^{\oplus}$ HTB-20 $^{\mathrm{Ts}}$, luminal B) were cultivated in Hybri-Care Medium (ATCC), 10\% FBS, $1.5 \mathrm{~g} / \mathrm{l} \mathrm{NaHCO}_{3}$ (Sigma Aldrich). MCF-7 (ATCC ${ }^{\circ}$ HTB-22 $2^{\text {тs }}$, HR-positive) were cultivated in DMEM, 10\% FBS, 2 mM L-glutamine. MDA-MB-231 (ATCC HTB-26 ${ }^{\mathrm{Tm}}$, TNBC, mesenchymal-like) were cultivated in DMEM, 10\% FBS, 2 mM L-glutamine. SKBR3 (ATCC HTB-30 ${ }^{\mathrm{ma}}$, HER2-positive) were cultivated in McCoy's 5a Modified Medium (Thermo Fisher Scientific Inc.), 10\% FBS. MDA-MB-468 (ATCC ${ }^{\oplus}$ HTB-132 $^{\mathrm{Tm}}$, TNBC, basal-like) were cultivated in DMEM/F12 Medium (Biochrom), 10\% FBS. T-47D (ATCC ${ }^{\bullet}$ HTB-133 $^{\mathrm{Tm}}$, Luminal A) were cultivated in RPMI-1640 (Thermo Fisher Scientific Inc.), 10\% FBS.

\section{Preparation of cell culture supernatants and cell pellets for ELISA and qPCR}

The different cells were seeded at a density of 40,000 cells per 12-well into four wells. For BCC, HMEC and MES, collagen-coating was performed in advance. At a confluency of $95 \%$, two wells were used for RNA isolation and to prepare the supernatants as conditioned media (CM).

For later RNA isolation, the wells were washed with PBS and the cells detached using Accutase ${ }^{\circ}$ (Sigma-Aldrich, St. Louis, MO, USA) and the pellet directly frozen in liquid nitrogen. For CM, the cells were washed with PBS once and each well filled with $800 \mu$ l corresponding medium without FBS or supplements of animal origin (e.g. bovine pituitary extract). ADSCs and epithelial cells received their basal medium; MES received EpiCult ${ }^{\mathrm{Tm}}-\mathrm{C}$ solely supplemented with L-glutamine and hydrocortisone. After $24 \mathrm{~h}$ the medium was frozen at $-80{ }^{\circ} \mathrm{C}$ for later ELISA-measurements.

Breast (cancer) cell lines were handled similarly and the CM obtained from the corresponding media without FBS.

\section{Adipogenic differentiation of ADSCs}

Adipogenic differentiation of the ADSCs was achieved using a mesenchymal stem cell adipogenic differentiation medium (PELOBIOTECH GmbH, Planegg/Martinsried, 
Germany) according to the manufacturer's protocol. ADSCs were seeded at a density of 40,000 cells per 12-well into four wells. Two wells were used for adipogenic differentiation, two as control. After reaching full confluency in the standard culture medium, the kit's media were used for 20 days (the controls were cultivated in the standard culture medium), followed by the preparation of CM and isolation of RNA.

\section{RNA isolation and real-time qPCR}

RNA was isolated using QIAGEN's RNeasy Mini Kit together with the QIAshredder (QIAGEN, Hilden, Germany) according to the manufacturer's protocols.

RNA concentration and quality was determined using a NanoDrop ND1000 Spectrophotometer (Thermo Fisher Scientific). Complementary DNA was synthesized with an oligo-dT primer and the SCRIPT cDNA Synthesis Kit (Jena Bioscience, Jena, Germany). A control sample without reverse transcriptase was carried along. Real-time PCR measurements were conducted with the SensiFAST ${ }^{\mathrm{mm}}$ $\mathrm{SYBR}^{\circ}$ No-ROX Kit (Bioline, London, UK) in triplicates for 40 cycles $\left(95^{\circ} \mathrm{C} 5 \mathrm{~s}, 60^{\circ} \mathrm{C} 10 \mathrm{~s}, 72^{\circ} \mathrm{C} 10 \mathrm{~s}\right)$ in a CFX Connect qPCR System (Bio-Rad Laboratories, Hercules, CA, USA). Detected transcript levels were normalized to the housekeeping gene hypoxanthine-guanine phosphoribosyltransferase (HPRT) using the $2^{-\triangle \mathrm{Cq}}$ method [28]. The primer sequences listed in Table 1 were used.

Fluorometric measurement of cytosolic free calcium levels For the measurement of cytosolic free calcium levels, 20,000 cells were seeded into 96-well cell culture plates 2 days before measurement. Then, they were washed twice with Hank's balanced salt solution (HBSS) containing 0.2\% BSA and $10 \mathrm{mM}$ HEPES (Sigma-Aldrich). Cells were incubated with $10 \mu \mathrm{M}$ Fura-2 AM in HBSS containing BSA, HEPES and 0,25\% pluronic acid (Sigma-Aldrich) for $60 \mathrm{~min}$ at $37^{\circ} \mathrm{C}$, were washed twice and $100 \mu \mathrm{l}$ HBSS+HEPES+BSA were added to each well. Analyses were performed in a microplate fluorometer with integrated pipetting system (BMG Labtech NOVOstar, Offenburg, Germany) at $340 \mathrm{~nm}$ resp. $380 \mathrm{~nm}$ for excitation and $510 \mathrm{~nm}$ for emission. Cells were allowed to adapt to $37^{\circ} \mathrm{C}$ for $10 \mathrm{~min}$ before LPA 18:1 (Avanti Polar Lipids Inc., Alabaster, AL, USA), solved in PBS containing $0.2 \%$ human serum albumin in indicated concentrations, was added. In cell line experiments, Ki16425 (Sigma-Aldrich) was used as LPA receptor antagonist in concentrations of 0,2 , or $20 \mu \mathrm{M} 1 \mathrm{~min}$ prior to the stimulation with LPA. The change in cytosolic free calcium $\left(\left[\mathrm{Ca}^{2+}\right]_{\mathrm{i}}\right)$ was calculated by dividing the measured fluorescence intensity at $510 \mathrm{~nm}$ with different excitation wavelengths (ratio $340 \mathrm{~nm} / 380 \mathrm{~nm}$ ). The baseline levels were subtracted. Adenosine triphosphate was used as a positive control, PBS with $0.2 \%$ human serum albumin as a negative control.

\section{ELISA measurements}

Levels of secreted ATX in the CM were detected by a highly sensitive ELISA Kit (Human ENPP-2/Autotaxin DuoSet ELISA in combination with DuoSet ELISA Ancillary Reagent Kit 2, R\&D Systems, Minneapolis, MN, USA) according to the manufacturer's instructions using the NOVOstar.

\section{Transmigration assay}

For the transmigration, Boyden chambers of PET-membrane transwell inserts (ThinCert ${ }^{\mathrm{Tw}}$, Greiner Bio-One GmbH, Frickenhausen, Germany) with a growth surface of $33.6 \mathrm{~mm}^{2}$ and a pore size of $8 \mu \mathrm{m}$ were used. The transwells were transferred into a 24-well plate and seeded with 50,000 cells in $300 \mu \mathrm{l}$ in duplicates with reduced Mammary Epithelial Cell Medium (containing 5\% of the normal supplement amount) or Epicult-C solely supplemented with L-glutamine and hydrocortisone.

The lower chamber was filled with the corresponding medium supplemented to either $1 \mu \mathrm{M}$ or $0.1 \mu \mathrm{M}$ LPA 18:1 dissolved in $0.2 \%$ fatty acid free BSA (Sigma) in PBS. A negative control without LPA but with BSA was also performed.

After an incubation period of $8 \mathrm{~h}$ at $37{ }^{\circ} \mathrm{C}$ and $5 \% \mathrm{CO}_{2}$, the transwells were washed, fixed with ice-cold methanol $(10 \mathrm{~min})$ and stained with DAPI $(1 \mu \mathrm{g} / \mathrm{ml}, 10 \mathrm{~min})$ (Life

Table 1 List of primers

\begin{tabular}{lll}
\hline & Forward Primer $\left(5^{\prime}->3^{\prime}\right)$ & Reverse Primer $\left(5^{\prime}->3^{\prime}\right)$ \\
\hline Human HPRT & AGTTCTGTGGCCATCTGCTT & GGCTTTGTATTTTGCTTTTCCAGT \\
Human LPAR1 & CCTGCAGTGCTTTATCGGA & TCAGATGGTCAGGAACGCTG \\
Human LPAR2 & CTCGGCATAGTTCTGGACCC & TTCTCATAGGCCAGCACGTC \\
Human LPAR3 & TACCGAGAGACCACGCTCAG & GCCTAAACCATCCAGGAGCA \\
Human LPAR4 & CCGCATACAAGTGGGTCCAT & GGGGTCCAGCATACCACAAA \\
Human LPAR5 & AAAGATCATGTACCCAATCACCTT & CTTAAACAGGGACTCCATTCTGAT \\
Human LPAR6 & CGCCATCTTCCAGATGAAC & TAGCGGTCCACGTTGATG \\
\hline
\end{tabular}


Technologies, Carlsbad, CA, USA). The cells in the inner part of the transwells were carefully wiped with a cotton swab to remove the non-migrated cells. Cells that migrated through the membrane were counted in 4 regions of interest (ROIs) per transwell (1 image per quadrant) in 40-fold magnification (Olympus IX83, cellSens Software, Olympus Corporation, Tokio, Japan). Quantification was performed semi-automatically using Fiji Is Just ImageJ (Fiji) 1.51u [29], an extended distribution of ImageJ.

As a comparison, the transmigration assay was also performed with the breast (cancer) cell lines. Here, Ki16425 $(2 \mu \mathrm{M})$ was used as LPA receptor antagonist in experiments with MDA-MB-231 as well as MDA-MB468. The assay itself was performed in reduced media without FBS.

\section{Statistics}

All the experiments were performed with $n=4$ except for the calcium imaging of the cell lines $(n=3)$ and the ATX ELISA and the qPCR of the individual cell lines $(n=1)$. Error bars indicate SD. Statistical analysis was performed using SPSS v.21.0 software (SPSS Software/ IBM, Armonk, NY, USA). Differences between groups were analyzed using the Kruskal-Wallis $\mathrm{H}$ test followed by a Mann-Whitney $U$ test for post-hoc analysis; the asymptotic significance was used. Significant $p$-value was set to $<0.05$ and corrected with the Bonferroni correction. All figures were created with GraphPad Prism 7.00 (GraphPad Software, La Jolla, CA, USA).

\section{Results}

Different mammary cells respond to stimulation with LPA 18:1 but express a different LPAR profile

We successfully isolated the distinct primary cell fractions of each patient's mammary tissue. ADSCs were isolated from tumor-adjacent (ADSC ta) and tumor-distant (ADSC td) or healthy (ADSC h) mammary fat tissue; epithelial and mesenchymal cells were isolated from mammary glands (HMEC, MES h) or mammary tumors (BCC, MES t).

In order to evaluate whether any of these cells may play a role in the ATX-LPA axis, their LPA receptor mRNA profile was analyzed and their calcium release after stimulation with LPA was measured. qPCR results (Fig. 1a) comparing the mRNA expression of LPAR to $H P R T$ indicated that ADSCs as well as MES mainly expressed LPAR1 and to a lesser extent LPAR6. Epithelial cells had a different expression profile of mainly LPAR6 but also LPAR1, LPAR2, and to a lesser extent LPAR3. LPAR4 and LPAR5 were not expressed by any of the cells. Although there were patient-dependent variations, there was no difference between cells from tumors and healthy origin.
Simulating the role of mature adipocytes in this context, ADSCs were differentiated into the adipogenic lineage. Their LPAR mRNA profile did not change considerably compared to the controls (Fig. 1b) but the expression of LPAR1 was reduced marginally. Again, there was no expression difference between cells from tumors and healthy tissue or tumor-distant and tumor-adjacent ADSCs.

Calcium imaging was used to determine the effect of LPA 18:1 on cytosolic free calcium levels. After stimulation with LPA, all different cell types released $\mathrm{Ca}^{2+}$ into the cytosol (Fig. 1c). A concentration of $0.01 \mu \mathrm{M}$ LPA 18:1 was sufficient to trigger a cellular response. The cytosolic free calcium levels also increased in a dosedependent manner with increasing LPA concentrations of up to $100 \mu \mathrm{M}$. Epithelial cells responded to increasing LPA concentrations with a higher calcium release than MES or ADSC. This effect was significant at concentrations of $1 \mu \mathrm{M}$ LPA and above $(p<0.0167)$. On a logarithmic scale the response of ADSCs was almost linear whereas especially healthy mammary epithelial cells and BCC, EpCAM-positive breast cancer cells, but also MES seemed to have a more sigmoidal nonlinear regression fit (plotted using GraphPad 7.00; nonlinear fit, variable slope). There was no significant difference between cells from tumors and healthy tissue.

MCF-10A expressed mainly LPAR6, BT-474 and T-47D expressed little amounts of LPAR1, MCF-7, SKBR3, and MDA-MB-468 expressed even smaller amounts of $L P A R 2$, while MDA-MB-231 expressed a litthe of LPAR2 and LPAR6 compared to HPRT. All breast (cancer) cell lines responded to $1 \mu \mathrm{M}$ LPA 18:1 with increasing cytosolic free calcium levels, too (Fig. 3c). T-47D had the weakest response with no reducing effect of the LPAR antagonist Ki16425. All the other cell lines showed a reduction of the calcium response with increasing concentrations of Ki16425.

\section{Cell type specific differences in ATX production of mammary cells}

An ATX-protein ELISA and qPCR for ATX mRNA levels were performed to analyze the expression level of ATX by different mammary cells. On average, ADSCs have a significantly higher ATX expression than MES on RNA level and a significantly higher expression than epithelial cells on RNA and protein level $(p<0.0167)$. MES also have a significantly higher ATX expression than epithelial cells both on RNA and protein level $(p<0.0167)$. Epithelial cells have almost no mRNA and protein expression of ATX (Fig. 2a). Again, strong differences between patients could be observed. There was no significant difference between cells from tumors and healthy tissue. Breast (cancer) cell lines did not express ATX, neither on RNA nor on protein level. 


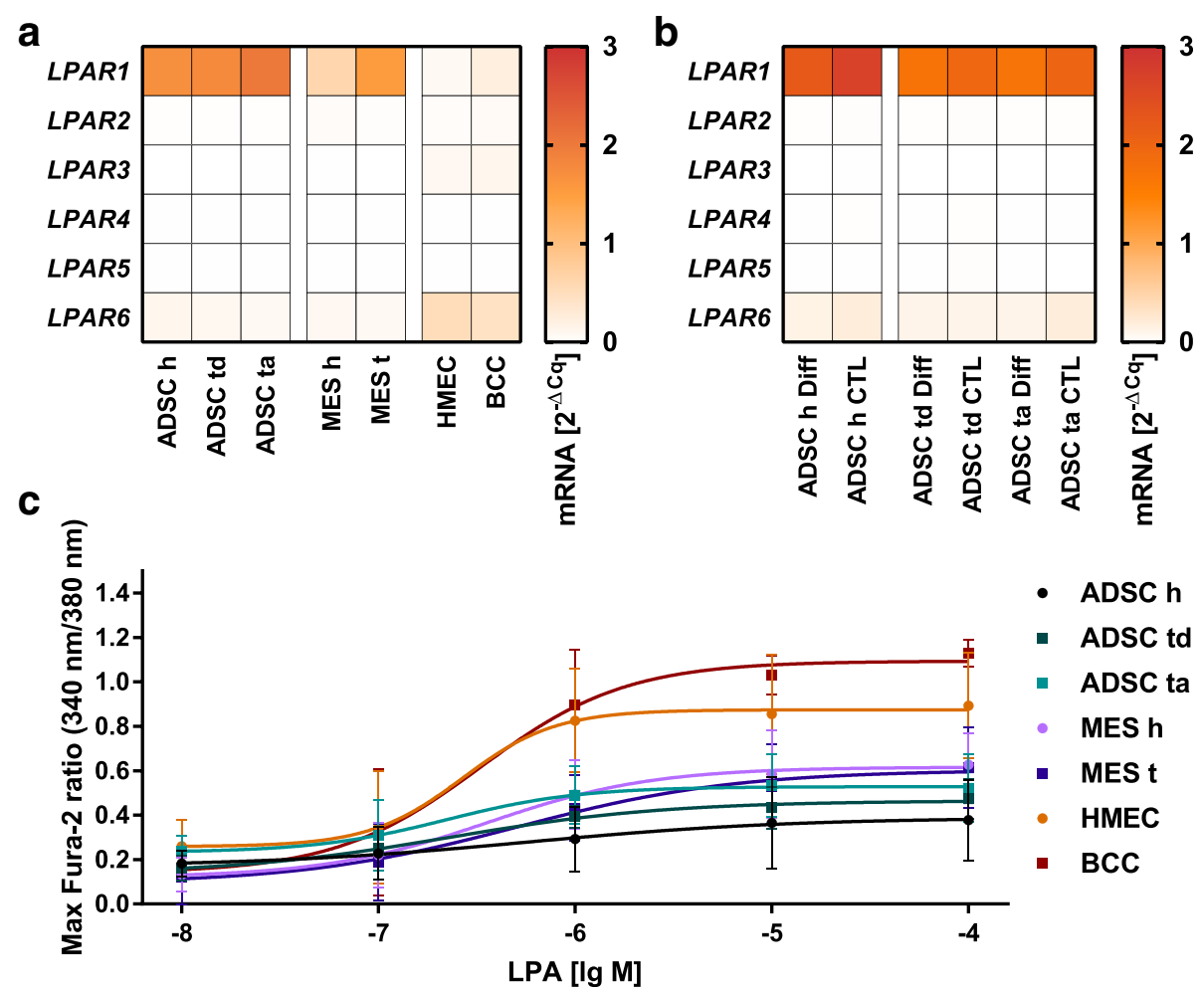

Fig. 1 Different mammary cells respond to stimulation with LPA 18:1 but express a different LPAR profile: (a/b) Mean relative LPAR mRNA expression. Values are calculated using the $2^{-\triangle C a}$ method, the reference is HPRT; (a) LPAR mRNA profile of ADSCs, MES and HMEC/BCC from tumors and healthy tissue; (b) The effect of adipogenic differentiation (Diff) on the LPAR mRNA profile of ADSCs compared to the control (CTL). (c) The effect of LPA on cytosolic free calcium levels; $x$-axis shows the molarity of LPA 18:1 in a common logarithmic scale, $y$-axis shows the maximal ratio of the emission of Fura-2 $(340 \mathrm{~nm} / 380 \mathrm{~nm}$ excitation). The mean measuring points with the SD are plotted and connected with a nonlinear fit (variable slope) using GraphPad Prism 7.00, epithelial cells have a significantly higher calcium release than ADSCs and mesenchymal cells at concentrations of $1 \mu \mathrm{M}$ LPA and above (not plotted for clarity, $p<0.0167$, Kruskal-Wallis $\mathrm{H}$ test, Mann-Whitney $\mathrm{U}$ test with Bonferroni correction); abbreviations: healthy h, tumor-distant td, tumor-adjacent ta, tumor t, EpCAM-positive breast cancer cells BCC; $n=4$

The ATX expression of ADSCs was analyzed after adipogenic differentiation (Fig. 2b) to compare them to mature adipocytes. In cells of healthy origin the adipogenic differentiation led to a significantly reduced expression of ATX on mRNA and protein level $(p<0.05)$. We observed higher variations between the tumor patients. Especially in differentiated tumor-adjacent ADSCs there was one patient with a more than fivefold expression. Compared to the controls, the average mRNA and protein ATX expression of differentiated ADSCs of tumor patients was higher than of healthy tissue.

Overall, ELISA and qPCR correlated with a Pearson correlation coefficient of 0.73 .

The transmigration of different mammary cells and breast cancer cell lines was stimulated by LPA

A transmigration assay was performed (Fig. 3a) to evaluate the effect of LPA 18:1 on the transmigration of primary mesenchymal and epithelial mammary cells.

Both mesenchymal cells of tumors and healthy origin were significantly stimulated by 0.1 and $1 \mu \mathrm{M}$ LPA, compared to the control without LPA $(p<0.0167)$. This resulted in approximately $200 \%$ more transmigrated cells after $8 \mathrm{~h}$ on average. The transmigration of epithelial cells was reduced significantly by 0.1 and $1 \mu \mathrm{M}$ LPA, whereas the reduction was only significant at $1 \mu \mathrm{M} \mathrm{LPA}$ for BCC.

As a comparison, the same assay was performed with different breast (cancer) cell lines: MDA-MB-231 and MDA-MB- 468 were significantly stimulated by $0.1 \mu \mathrm{M}$ and $1 \mu \mathrm{M}$ LPA 18:1 in their transmigration rate. With the addition of Ki16425 there was no significant stimulation compared to the control without LPA. MCF-10A had a significant inhibition in their transmigration rate at $1 \mu \mathrm{M}$ LPA. The other breast cancer cell lines (BT-474, MCF-7, SKBR3, and T-47D) were not stimulated by LPA.

\section{Discussion}

The role of the ATX-LPA axis in different cancers has been subject to an increasing number of publications within the recent past. Previous studies have shown that 

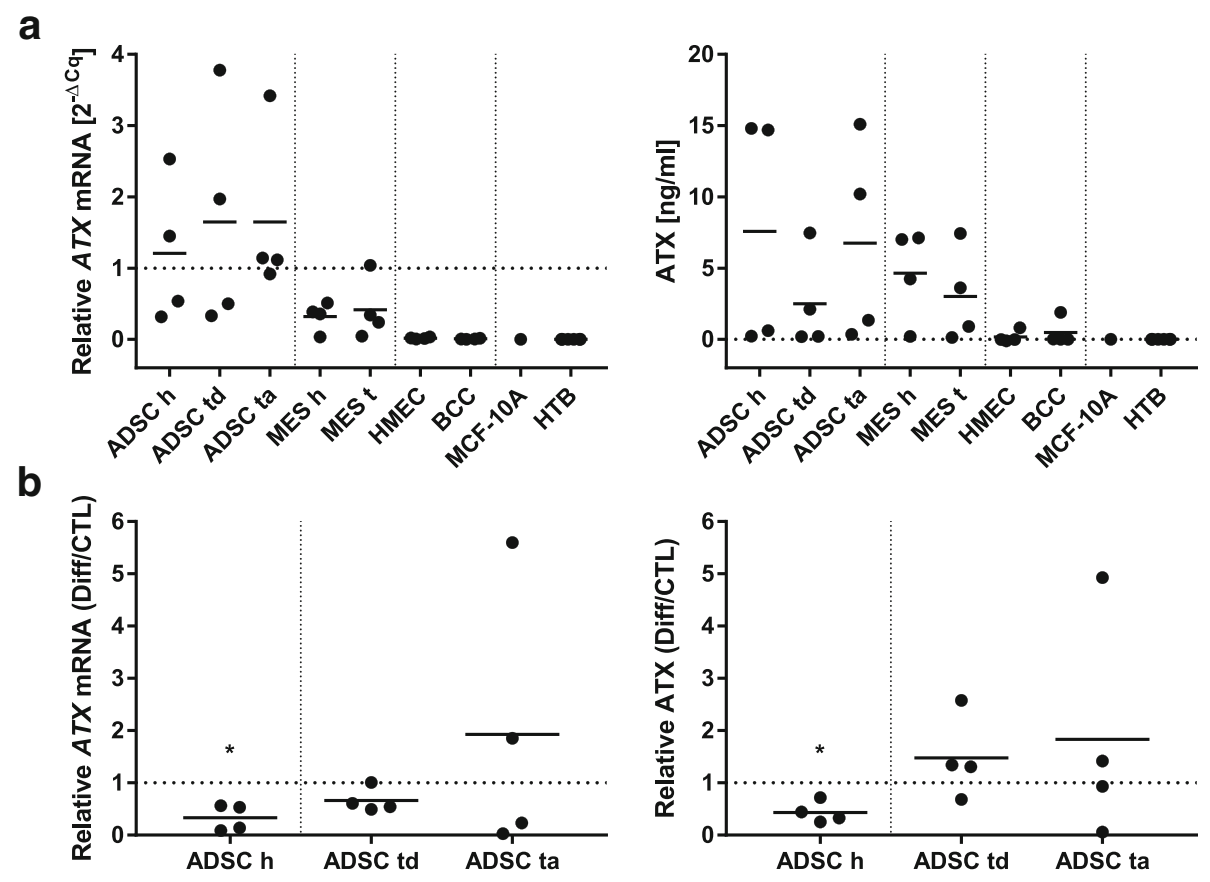

Fig. 2 Cell type specific differences in ATX production of mammary cells: (a) The expression of ATX on mRNA and protein level by different mammary cells; left: relative ATX mRNA levels compared to HPRT (y-axis) of different cell types ( $x$-axis); right: ATX protein in the conditioned media in $\mathrm{ng} / \mathrm{ml}$ (y-axis) of different cell types (X-axis); ADSCs have a significantly higher ATX expression than MES on RNA level and a significantly higher expression than epithelial cells on RNA and protein level, MES also have a significantly higher ATX expression than epithelial cells both on RNA and protein level (not plotted for clarity, $\mathrm{p}<0.0167$, Kruskal-Wallis $\mathrm{H}$ test, Mann-Whitney $\mathrm{U}$ test with Bonferroni correction); (b) The effect of adipogenic differentiation on the ATX expression; left: relative ATX mRNA expression, right: relative ATX protein expression, $x$-axis shows the cell type, $y$-axis shows the ratio of differentiation (Diff) and control (CTL); the biological samples (dots) and the average (line) are plotted; abbreviations: healthy $h$, tumor-distant td, tumor-adjacent ta, tumor t, EpCAM-positive breast cancer cells BCC, HTB human tumor bank; $n=4$ (MCF-10A $n=1, \mathrm{HTB} n=6)$, ${ }^{*} p<0.05$, Kruskal-Wallis $\mathrm{H}$ test, Mann-Whitney $\mathrm{U}$ test with Bonferroni correction

in breast cancers not the tumor but the surrounding fat tissue produces ATX [19]. To further investigate this phenomenon on a cellular level, we isolated different cell populations of breast cancer and healthy tissue and compared the results to established breast (cancer) cell lines.

Benesch et al. [19] described a vicious cycle in tumors secreting inflammatory cytokines which then stimulate fat tissue to produce ATX leading to more LPA. LPA again stimulates the tumor to proliferate and produce more cytokines. All cell types analyzed in the present investigation responded to stimulation with LPA 18:1 in the calcium imaging assay (Fig. 1c). Therefore, the vicious cycle would have had effects on all the cell populations in our setting, even though not on the same level and through different LPA receptors (Fig. 1a/b). Mesenchymal cells and ADSCs expressed mainly LPAR1 and to some extent LPAR6 mRNA. Epithelial cells had the highest expression of LPAR6 and to a lesser extent LPAR1-3 mRNA. Depending on the breast tumor subtype, elevated LPAR3 mRNA levels have been reported. Especially HER2-positive, HR-negative, less differentiated tumors that are more aggressive are associated with increased LPAR3 levels [25]. We did not detect elevated LPAR3 levels in our luminal $\mathrm{B}(n=3)$ and $\operatorname{TNBC}(n=1)$ patients. LPAR4 and LPAR5 were not expressed by the mammary cell populations at all. In general, a correlation of the LPAR mRNA profile of our cells of tumors and healthy tissue was detected. While the mammary epithelial cell line MCF-10A had a similar LPAR profile to the primarily isolated cells with a high expression of LPAR6, the breast cell lines revealed strikingly varying $L P A R$ mRNA profiles (Fig. 3b). Compared to the housekeeping gene HPRT, the LPAR expression was much lower than in our primary cells. BT-474 and T-47D expressed little amounts of LPAR1, MCF-7, SKBR3, and MDA-MB-468 expressed even smaller amounts of LPAR2, while MDA-MB-231 expressed a little of LPAR2 and LPAR6 mRNA. This is contrary to previously published data [30] that reported MDA-MB-231 expressing higher amounts of LPAR1 than LPAR2 and more than 80 times higher than by MCF-10A [31]. For MCF-7, MDA-MB-468, and T-47D similar LPAR1-3 mRNA profiles have been reported [30]. It is possible that the cell lines with differing results mutated in laboratories over time. 

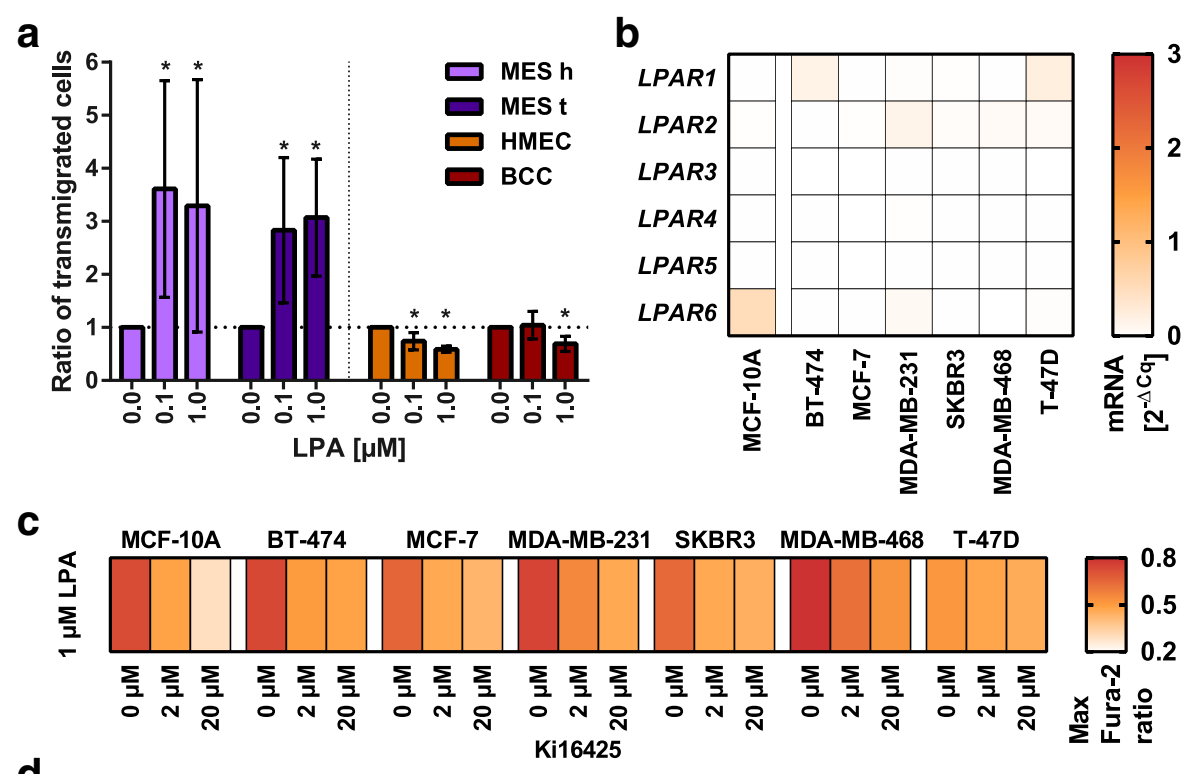

d

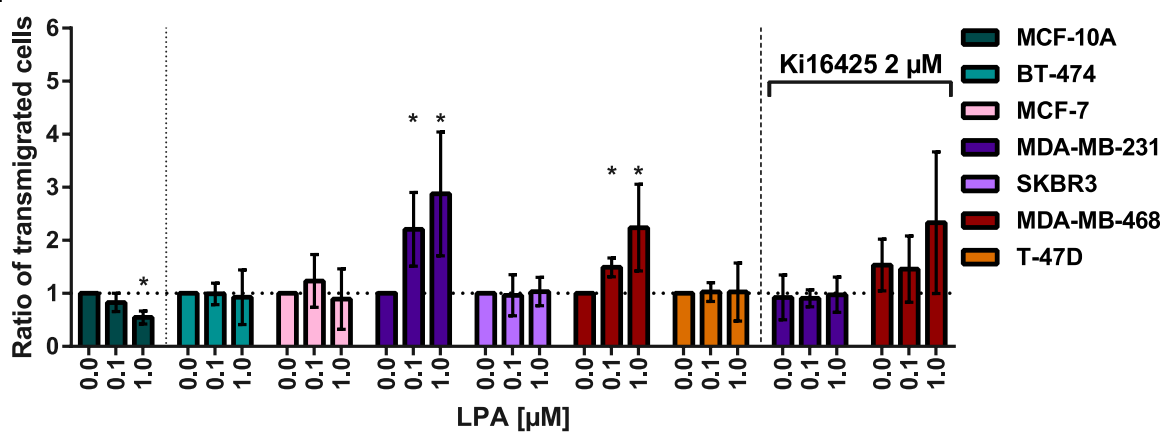

Fig. 3 The transmigration stimulated by LPA 18:1 and profiling of breast (cancer) cell lines: (a) The ratio of transmigrated cells compared to the control ( $y$-axis) in varying LPA concentrations ( $x$-axis) of mesenchymal and epithelial cells of healthy and tumor tissue, $n=4$; abbreviations: healthy $h$, tumor t, EPCAM-positive breast cancer cells BCC; (b) To HPRT relative LPAR mRNA expression of different breast (cancer) cell lines, $\mathrm{n}=1$; (c) Mean maximal ratio of the emission of Fura-2 $(340 \mathrm{~nm} / 380 \mathrm{~nm}$ excitation) of different breast (cancer) cell lines as effect of $1 \mu \mathrm{M}$ LPA on cytosolic free calcium levels with the addition of the LPAR antagonist Ki16425, $n=3$; (d) The ratio of transmigrated cells compared to the control ( $y$-axis) in varying LPA concentrations ( $x$-axis) of different breast (cancer) cell lines $(n=4)$ with or without the addition of $2 \mu M$ Ki16425; plotted are the means and their SD; ${ }^{*} p<0.0167$, Kruskal-Wallis $\mathrm{H}$ test, Mann-Whitney $\mathrm{U}$ test with Bonferroni correction

Interestingly, ADSCs showed an almost linear increase in cytosolic free calcium levels despite of an exponential increase in the stimulation reagent LPA 18:1 (Fig. 1c). We could see typical sigmoidal curves in our EpCAM-positive epithelial tumor cells (named BCC), in our healthy human mammary epithelial cells, and with a weaker response also in mesenchymal cells. We stimulated with a wide range of concentrations that included and exceeded the physiological plasma concentrations of LPA [32]. It is possible, that ADSCs have their plateau phase in their calcium release at even higher LPA concentrations. The breast (cancer) cell lines were also stimulated in the assay. The LPAR antagonist Ki16425 was used in increasing concentrations to evaluate possible inhibitory effects on the cell lines. In general, the cell lines showed comparable results. While $1 \mu \mathrm{M}$ LPA stimulated the cells, increasing concentrations of Ki16425 reduced the response. Not only was the stimulation of T-47D by LPA relatively weak, also the possibility to reduce the calcium response using Ki16425 seemed to be limited. During adipogenic differentiation, we were able to detect patient-dependent differences in the amount of accumulated triglycerides and hence differences in the differentiation potential. This may also be an effect of the patient's age [33] or physiology. While other groups reported that LPAR1 is the most abundant subtype in adipose tissue and showed a decrease of LPAR1 expression with adipogenic differentiation [34], we also found that ADSCs and adipocytes highly expressed LPAR1, however, we detected only a marginal reduction of LPAR1 mRNA expression after differentiation.

We could show that especially ADSCs and not the mammary tumor cell populations expressed high levels of ATX (Fig. 2a). Epithelial cells seem to be responder in the ATX-LPA axis. In experiments with murine cell 
lines it was shown that differentiating preadipocytes increases the expression of ATX [18]. In contrast, undifferentiated human ADSCs produced significantly $(p<$ 0.5) more ATX than differentiated adipocytes of healthy origin (Fig. 2b). In tumor patients this seemed to be reversed. In one tumor patient we even measured a higher expression of ATX after adipogenic differentiation. It is possible that this is not just a patient variance but a dysregulation as a result of the tumor's influence and part of the tumor malignancy. Variances between different breast tumor types are also likely as e.g. also LPAR3 levels can differ significantly [25]. With no expression of ATX on RNA and protein level, the different cell lines behave like primarily isolated epithelial cells. This further supports the thesis that breast tumors do not express ATX. With a Pearson correlation coefficient of 0.73, qPCR and ELISA results of the ATX expression correlate relatively high. This indicates a good reliability of the used methods.

Concerning the effect of elevated ATX and LPA levels on higher metastasis rates, we performed transmigration assay with our cells. LPA has already been linked to increased fibroblast migration [35] as well as tumor cell migration and invasion in breast cancer cell lines [36]. Here, we evaluated the effect of LPA 18:1 on the transmigration of primary mesenchymal and epithelial mammary cells. Mesenchymal cells were stimulated in their transmigration whereas epithelial cells are inhibited. The reason can be that they signal LPA through different LPARs. Their mRNA expression profile suggests that they signal mainly via LPAR1 or LPAR6. We could not detect differences between cells isolated from tumors to healthy cells. Maybe LPA does not directly stimulate their transmigration but their proliferation or their secretion of inflammatory cytokines. Evaluating these factors should be subject of further research. When comparing the primarily isolated cells with different cell lines in their transmigration rate, it is interesting that the TNBC MDA-MB-231 and MDA-MB-468 were stimulated significantly $(p<0.0167)$ whereas the HER2-positive SKBR3, the luminal B BT-474, the luminal A MCF-7 and T-47D, and the non-cancerous MCF-10A seemed to react to LPA similar to our healthy and tumor epithelial cells with a non-stimulated or even inhibited transmigration. Our significant results showing the stimulation of MDA-MB-231 and MDA-MB-468 are also comparable to previous findings of collagen-coated transmigration assays although T-47D was not stimulated in our setting [30]. It is possible that the cells had problems in the serum-free assays, especially the long-lasting transmigration assay. The inhibitory effect of Ki16425 on MDA-MB-231 has also been subject to experiments in Matrigel-coated invasion assays [31] with similar results. With Ki16425 used as a receptor antagonist, neither MDA-MB-231 nor MDA-MB-468 were significantly stimulated by LPA 18:1 anymore. The transmigration rate of MDA-MB-231 was at the normal level with the addition of Ki16425 while it tended to be elevated for MDA-MB-468 even though not significantly.

From these results we conclude that the transmigration of tumor cells and its possibility to be stimulated by LPA 18:1 most probably depends on the tumor subtype.

Besides the effect of LPA on tumor cytokine expression or proliferation and the use of inhibitory reagents such as Ki16425, the stimulatory effect of radiotherapy on the ATX expression [20], and associated resistances to chemotherapeutics, should be studied on a cellular level. The combined knowledge will be of importance to further assess the ATX-LPA-axis as potential target for cancer therapy.

\section{Conclusion}

The fat tissue is the main source of ATX and LPA in breast tissue. ADSCs and adipocytes contribute to the ATX expression to different extents. In healthy patients ADSCs are predominant, in tumor patients adipocytes could prevail. We demonstrated that the isolated mammary cell populations respond to LPA with a varying receptor profile. LPA stimulated the transmigration of primary mesenchymal breast cells whereas epithelial cells were inhibited without any differences between tumorous and healthy tissue. In comparison, LPA stimulated solely the two established TNBC tumor cell lines. Therefore, targeting the ATX-LPA axis may represent an additive cancer therapy for some invasive and metastatic breast tumors depending on their subtype.

\section{Abbreviations}

ADSC: Adipose-derived stem cell; ATX: Autotaxin; BCC: EpCAM-positive cells; BSA: Bovine serum albumin; CM: Conditioned media; CTL: Control;

Diff: Adipogenic differentiation; h: Healthy; HBSS: Hank's balanced salt solution; HMEC: Human mammary epithelial cells; HPRT: Hypoxanthine-guanine phosphoribosyltransferase; HTB: Human tumor bank; LPA: Lysophosphatidic acid; LPAR: LPA receptor; LPC: Lysophosphatidylcholine; MES: Mesenchymal cells; ROI: Region of interest; ta: Tumor-adjacent; td: Tumor-distant; TNBC: Triple-negative breast cancer

\section{Acknowledgements}

The authors are grateful and thank all patients who participated in this study. We would like to thank Prof. Dr. Arndt Hartmann, Institute of Pathology, and Prof. Dr. Matthias W. Beckmann, Department of Obstetrics and Gynaecology, University Hospital of Erlangen, for their support in acquiring donors, Mareike Wittenberg for providing us with the cell lines, Katharina Kalb, Stefan

Fleischer, and Ilse Arnold-Herberth for their excellent technical support and all clinical staff members for providing us with the tissue for cell isolations.

\section{Funding}

This study was funded by the interdisciplinary center for clinical research (IZKF), University of Erlangen-Nürnberg to A.E.K., the German Research Foundation (KR4391/1-1), ELAN-Fonds (13-12-11-1-Weigand), University of Erlangen-Nürnberg, the Sofie-Wallner Stiftung, the Forschungsstiftung Medizin, University of Erlangen-Nürnberg, the Marohn-Stiftung, University of Erlangen-Nürnberg, the Manfred Roth Foundation, and the Xue Hong and Hans Georg Geis Foundation. The funding bodies did not have any influence on the design of the study, the collection, analysis, and interpretation of data, or the writing of the manuscript. 


\section{Availability of data and materials}

The datasets generated and analyzed during the current study are available from the corresponding author on reasonable request.

\section{Authors' contributions}

RS, KW, JWR, SS, PLS, ReSt, and MR performed research and analyzed data; PAF and REH analyzed data; RS, KW, JWR, AEK, AMB, and AW designed research; RS and KW wrote the paper. All authors read and approved the final manuscript.

\section{Ethics approval and consent to participate}

Human tissue collection was approved by the Ethics Committee of the University of Erlangen-Nürnberg (Ethics \#264_13B, \#99_15 BC) in accordance with the World Medical Association Declaration of Helsinki. Written informed consent was obtained from all patients.

\section{Consent for publication}

Not applicable

\section{Competing interests}

The authors declare that they have no competing interests.

\section{Publisher's Note}

Springer Nature remains neutral with regard to jurisdictional claims in published maps and institutional affiliations.

\section{Author details}

'Department of Plastic and Hand Surgery and Laboratory for Tissue Engineering and Regenerative Medicine, University Hospital of Erlangen, Friedrich-Alexander-University Erlangen-Nürnberg (FAU), Krankenhausstr. 12, 91054 Erlangen, Germany. ${ }^{2}$ Department of Medicine I, University Hospital of Erlangen, Friedrich-Alexander-University Erlangen-Nürnberg (FAU), Erlangen, Germany. ${ }^{3}$ Laboratory for Molecular Medicine, Department of Gynecology and Obstetrics, University Hospital of Erlangen, Friedrich-Alexander-University Erlangen-Nürnberg (FAU), Erlangen, Germany. ${ }^{4}$ Department of Gynecology and Obstetrics, Comprehensive Cancer Center Erlangen ER-EMN, University Hospital Erlangen, Friedrich-Alexander-University Erlangen-Nürnberg (FAU), Erlangen, Germany.

Received: 6 June 2018 Accepted: 30 November 2018

Published online: 19 December 2018

\section{References}

1. Siegel RL, Miller KD, Jemal A. Cancer statistics, 2017. CA Cancer J Clin. 2017; 67(1):7-30.

2. Kawalec $P$, Lopuch S, Mikrut A. Effectiveness of targeted therapy in patients with previously untreated metastatic breast cancer: a systematic review and meta-analysis. Clinical breast cancer. 2015;15(2):90-100 e101.

3. Schwirzke M, Evtimova V, Burtscher $H$, Jarsch $M$, Tarin D, Weidle UH Identification of metastasis-associated genes by transcriptional profiling of a pair of metastatic versus non-metastatic human mammary carcinoma cell lines. Anticancer Res. 2001;21(3B):1771-6.

4. Stracke ML, Krutzsch HC, Unsworth EJ, Arestad A, Cioce V, Schiffmann E, Liotta LA. Identification, purification, and partial sequence analysis of autotaxin, a novel motility-stimulating protein. J Biol Chem. 1992;267(4): 2524-9.

5. Goding JW, Grobben B, Slegers H. Physiological and pathophysiological functions of the ecto-nucleotide pyrophosphatase/phosphodiesterase family. Biochim Biophys Acta. 2003;1638(1):1-19.

6. Umezu-Goto M, Kishi Y, Taira A, Hama K, Dohmae N, Takio K, Yamori T, Mills GB, Inoue K, Aoki J, et al. Autotaxin has lysophospholipase D activity leading to tumor cell growth and motility by lysophosphatidic acid production. J Cell Biol. 2002;158(2):227-33.

7. Tokumura A, Majima E, Kariya Y, Tominaga K, Kogure K, Yasuda K, Fukuzawa K. Identification of human plasma lysophospholipase D, a lysophosphatidic acid-producing enzyme, as autotaxin, a multifunctional phosphodiesterase. J Biol Chem. 2002;277(42):39436-42.

8. Hecht JH, Weiner JA, Post SR, Chun J. Ventricular zone gene-1 (vzg-1) encodes a lysophosphatidic acid receptor expressed in neurogenic regions of the developing cerebral cortex. J Cell Biol. 1996;135(4):1071-83.
9. An S, Bleu T, Hallmark OG, Goetzl EJ. Characterization of a novel subtype of human $\mathrm{G}$ protein-coupled receptor for lysophosphatidic acid. J Biol Chem. 1998:273(14):7906-10.

10. Bandoh K, Aoki J, Hosono H, Kobayashi S, Kobayashi T, Murakami-Murofushi K, Tsujimoto M, Arai H, Inoue K. Molecular cloning and characterization of a novel human G-protein-coupled receptor, EDG7, for lysophosphatidic acid. J Biol Chem. 1999;274(39):27776-85.

11. van Meeteren LA, Moolenaar WH. Regulation and biological activities of the autotaxin-LPA axis. Prog Lipid Res. 2007:46(2):145-60.

12. Moolenaar WH. Bioactive lysophospholipids and their $\mathrm{G}$ protein-coupled receptors. Exp Cell Res. 1999;253(1):230-8.

13. Aikawa S, Kano K, Inoue A, Aoki J. Proliferation of mouse endometrial stromal cells in culture is highly sensitive to lysophosphatidic acid signaling. Biochem Biophys Res Commun. 2017;484(1):202-8.

14. Quan M, Cui JJ, Feng X, Huang Q. The critical role and potential target of the autotaxin/lysophosphatidate axis in pancreatic cancer. Tumour Biol. 2017;39(3):1010428317694544.

15. Zhang R, Wang J, Ma S, Huang Z, Zhang G. Requirement of Osteopontin in the migration and protection against Taxol-induced apoptosis via the ATXLPA axis in SGC7901 cells. BMC Cell Biol. 2011;12:11.

16. Kehlen A, Englert N, Seifert A, Klonisch T, Dralle H, Langner J, Hoang-Vu C. Expression, regulation and function of autotaxin in thyroid carcinomas. Int J Cancer. 2004;109(6):833-8.

17. D'Souza K, Kane DA, Touaibia M, Kershaw EE, Pulinilkunnil T, Kienesberger PC. Autotaxin is regulated by glucose and insulin in adipocytes. Endocrinology. 2017;158(4):791-803.

18. Ferry G, Tellier E, Try A, Gres S, Naime I, Simon MF, Rodriguez M, Boucher J, Tack I, Gesta S, et al. Autotaxin is released from adipocytes, catalyzes lysophosphatidic acid synthesis, and activates preadipocyte proliferation. Up-regulated expression with adipocyte differentiation and obesity. J Biol Chem. 2003;278(20):18162-9.

19. Benesch MG, Tang X, Dewald J, Dong WF, Mackey JR, Hemmings DG, McMullen TP, Brindley DN. Tumor-induced inflammation in mammary adipose tissue stimulates a vicious cycle of autotaxin expression and breast cancer progression. FASEB J. 2015;29(9):3990-4000.

20. Meng G, Tang X, Yang Z, Benesch MGK, Marshall A, Murray D, Hemmings DG, Wuest F, McMullen TPW, Brindley DN. Implications for breast cancer treatment from increased autotaxin production in adipose tissue after radiotherapy. FASEB J. 2017;31(9):4064-77.

21. Zhang H, Xu X, Gajewiak J, Tsukahara R, Fujiwara Y, Liu J, Fells Jl, Perygin D, Parrill AL, Tigyi G, et al. Dual activity lysophosphatidic acid receptor pan-antagonist/autotaxin inhibitor reduces breast cancer cell migration in vitro and causes tumor regression in vivo. Cancer Res. 2009;69(13):5441-9.

22. Yang SY, Lee J, Park CG, Kim S, Hong S, Chung HC, Min SK, Han JW, Lee $H W$, Lee HY. Expression of autotaxin (NPP-2) is closely linked to invasiveness of breast cancer cells. Clin Exp Metastasis. 2002;19(7):603-8.

23. Benesch MG, Zhao YY, Curtis JM, McMullen TP, Brindley DN. Regulation of autotaxin expression and secretion by lysophosphatidate and sphingosine 1-phosphate. J Lipid Res. 2015;56(6):1134-44.

24. Brindley DN, Lin FT, Tigyi GJ. Role of the autotaxin-lysophosphatidate axis in cancer resistance to chemotherapy and radiotherapy. Biochim Biophys Acta. 2013;1831(1):74-85.

25. Popnikolov NK, Dalwadi BH, Thomas JD, Johannes GJ, Imagawa WT. Association of autotaxin and lysophosphatidic acid receptor 3 with aggressiveness of human breast carcinoma. Tumour Biol. 2012;33(6): 2237-43.

26. Liu S, Umezu-Goto M, Murph M, Lu Y, Liu W, Zhang F, Yu S, Stephens LC, Cui $X$, Murrow G, et al. Expression of autotaxin and lysophosphatidic acid receptors increases mammary tumorigenesis, invasion, and metastases. Cancer Cell. 2009;15(6):539-50.

27. Weigand A, Boos AM, Tasbihi K, Beier JP, Dalton PD, Schrauder M, Horch RE, Beckmann MW, Strissel PL, Strick R. Selective isolation and characterization of primary cells from normal breast and tumors reveal plasticity of adipose derived stem cells. Breast Cancer Res. 2016;18(1):32.

28. Livak KJ, Schmittgen TD. Analysis of relative gene expression data using real-time quantitative PCR and the $2(-$ Delta Delta $C(T))$ method. Methods. 2001;25(4):402-8.

29. Schindelin J, Arganda-Carreras I, Frise E, Kaynig V, Longair M, Pietzsch T, Preibisch S, Rueden C, Saalfeld S, Schmid B, et al. Fiji: an open-source platform for biological-image analysis. Nat Methods. 2012;9(7):676-82. 
30. Chen M, Towers LN, O'Connor KL. LPA2 (EDG4) mediates rho-dependent chemotaxis with lower efficacy than LPA1 (EDG2) in breast carcinoma cells. Am J Physiol Cell Physiol. 2007;292(5):C1927-33.

31. Li TT, Alemayehu M, Aziziyeh Al, Pape C, Pampillo M, Postovit LM, Mills GB, Babwah AV, Bhattacharya M. Beta-arrestin/Ral signaling regulates lysophosphatidic acid-mediated migration and invasion of human breast tumor cells. Mol Cancer Res. 2009; (7):1064-77.

32. Hosogaya S, Yatomi Y, Nakamura K, Ohkawa R, Okubo S, Yokota H, Ohta M, Yamazaki H, Koike T, Ozaki Y. Measurement of plasma lysophosphatidic acid concentration in healthy subjects: strong correlation with lysophospholipase D activity. Ann Clin Biochem. 2008;45(Pt 4):364-8.

33. Bajek A, Czerwinski M, Olkowska J, Gurtowska N, Kloskowski T, Drewa T. Does aging of mesenchymal stem cells limit their potential application in clinical practice? Aging Clin Exp Res. 2012;24(5):404-11.

34. Rancoule C, Dusaulcy R, Treguer K, Gres S, Attane C, Saulnier-Blache JS. Involvement of autotaxin/lysophosphatidic acid signaling in obesity and impaired glucose homeostasis. Biochimie. 2014:96:140-3.

35. Pietruck F, Busch S, Virchow S, Brockmeyer N, Siffert W. Signalling properties of lysophosphatidic acid in primary human skin fibroblasts: role of pertussis toxin-sensitive GTP-binding proteins. Naunyn Schmiedeberg's Arch Pharmacol. 1997;355(1):1-7.

36. Alemayehu M, Dragan M, Pape C, Siddiqui I, Sacks DB, Di Guglielmo GM, Babwah AV, Bhattacharya M. beta-Arrestin2 regulates lysophosphatidic acidinduced human breast tumor cell migration and invasion via Rap1 and IQGAP1PLoS One. 2013;8(2):e56174.

Ready to submit your research? Choose BMC and benefit from:

- fast, convenient online submission

- thorough peer review by experienced researchers in your field

- rapid publication on acceptance

- support for research data, including large and complex data types

- gold Open Access which fosters wider collaboration and increased citations

- maximum visibility for your research: over $100 \mathrm{M}$ website views per year

At $\mathrm{BMC}$, research is always in progress.

Learn more biomedcentral.com/submissions 